\title{
An Update of The Classification and \\ Treatment of Birthmarks in The Head and Neck Region
}

\author{
Baş ve Boyun Bölgesindeki Doğum Lekelerinin
}

Tedavi ve Sınıflamasında Güncelleme

Gürkan Kayabaşoğlu, Alpen Nacar, Mahmut Sinan Yılmaz, Mehmet Güven

Department Of Otorhinolaryngology, Head And Neck Surgery, Sakarya University Education And Training Hospital, Sakarya, Turkey

Keywords: Roll, planing; health developing; Urban sustainable development

Aplication: 03.10.2013 Accepted: 11.11.2013

\section{Introduction}

Birthmarks are present in $8-10 \%$ of the population. In the United States, of the 400,000 newborns who have a birthmark each year, $70 \%$ are misdiagnosed. ${ }^{1}$ Unfortunately most are diagnosed as hemangioma due to the clinical characteristics, findings, and varied physical appearances; as such, in this respect, patients are misinformed and misguided. Another issue is the use of incorrect terminology due to physicians not following updates in both nomenclature and class/grading schemes. In this review, we consider the misconceptions and confusing aspects of commonly occurring birthmarks and take into consideration the advances in updates to present this topic in a way that will be of use to most specialties.

\section{Classification}

Mulliken and Glowacki, in 1982, described a novel classification scheme for vascular anomalies on the basis of endothelial cells and clinical characteristics. This classification scheme separated vascular tumors from vascular malformations based on their clinical presentation, radio- logic, and pathologic attributes. 1 In 1996, the ISSVA (International Society for the Study of Vascular Anomalies) used the fundamentals of Mulliken and Glowacki's classification system to create a new classification system with the goal of establishing a universal convention with regard to the classification of vascular anomalies. Vascular tumors and vascular malformations were the two main categories. In 1999, Waner and Suen took the ISSVA classification and sought to clear up some ambiguities and created what was considered a more clinically useful classification system. ${ }^{2}$ (Table)

In the ISSVA's update of the classification system, aside from the morphologic and clinical differences, the immunohistochemical aspects played an important role in the categorization, and in biological studies since 1982 the apparent differences between tumors and malformations have been revealed. ${ }^{3}$

In considering the differential diagnosis for vascular tumors and vascular malformations, is not only important to assess the clinical, radiological and pathological fin- 
Table 1: Classification of vascular anomalies

\begin{tabular}{|c|c|c|c|}
\hline & Infantile hemangiomas & & \\
\hline & Congenital hemangiomas $(\mathrm{R})$ & $\mathrm{CH}, \mathrm{NICH})$ & \\
\hline & Tufted angioma (with or with & out Kasabah-Meritt Syndrom & \\
\hline & Kaposiform hemangioendoth & elioma (with or without Kasa & ah-Meritt Syndrome) \\
\hline & Spindle cell hemangioendoth & elioma & \\
\hline & $\begin{array}{l}\text { Dermatologic acquired vascu } \\
\text { glomeruloid hemangioma, } \mathrm{m}\end{array}$ & $\begin{array}{l}\text { lar tumors (Pyogenic granulo } \\
\text { crovenular hemangioma, etc }\end{array}$ & a, targeoid hemangioma, \\
\hline \multirow{13}{*}{$\begin{array}{l}\text { Vascular Malforma- } \\
\text { tions }\end{array}$} & \multirow{9}{*}{$\begin{array}{l}\text { Slow- flow vascular malfor- } \\
\text { mations }\end{array}$} & \multirow{3}{*}{$\begin{array}{l}\text { Capillary malformations } \\
\text { (CM) }\end{array}$} & Port-wine stain \\
\hline & & & Telengiectasia \\
\hline & & & Angiokeratoma \\
\hline & & \multirow{5}{*}{ Venous malformation (VM) } & Common sporadic VM \\
\hline & & & Bean syndrome \\
\hline & & & $\begin{array}{l}\text { Familial cutaneous and mu- } \\
\text { cosal venous malformation } \\
\text { (VMCM) }\end{array}$ \\
\hline & & & $\begin{array}{l}\text { Glomuvenousmalformation } \\
\text { (GVM) }\end{array}$ \\
\hline & & & Mafucci syndrome \\
\hline & & \multicolumn{2}{|l|}{ Lympatic malformation (LM) } \\
\hline & \multirow{3}{*}{ Fast-flow malformations } & \multicolumn{2}{|l|}{ Arterial malformation (AM) } \\
\hline & & \multicolumn{2}{|l|}{ Arteriovenous fistula (AVF) } \\
\hline & & \multicolumn{2}{|c|}{ Arteriovenous malformation (AVM) } \\
\hline & $\begin{array}{l}\text { Complex-combined vascular } \\
\text { malformations }\end{array}$ & \multicolumn{2}{|c|}{ CVM,CLM,LVM,CLVM,AVM-LM,CM-AVM } \\
\hline
\end{tabular}

dings, but also to consider the morbidity and treatment options. Vascular tumors are generally not seen at the time of birth but become visible within the first few weeks of life. Additionally, there is variation amongst the different classes of tumors: while some tumors (e.g. In- fantile hemangioma) can regress with time, others can persist (e.g. Kaposiform hemangioendothelioma). Furthermore, even amongst the subgroups, there can be subtypes that differ in their characteristics (e.g. Rapidly involuting congenital hemangioma versus non-involuting 
congenital hemangioma). Vascular malformations, on the other hand, even if asymptomatic, are often present at birth and can continue to grow commensurate with the growth of the child, and if not treated, can undergo rapid growth and lead to more widespread problems. ${ }^{4}$

In the pathology of hemangiomas, it has been shown that there is an rapid increase in the amount and proliferation rate of endothelial cells and increase in mast cells associated with angiogenesis (which return to normal level upon regression) and basal membrane multi-lamination can be seen. Vascular malformations, however, do not exhibit hyperplasia but are due to progressive ectasia in the vasculature, the basal lamina is covered by a flat and thin endothelium with no increase in mast cells. In larger hemangiomas, platelet half-life is reduced, platelets, along with other clotting factors, may become trapped within the tumor and lead to coagulopathy (KasabachMerritt phenomenon). In vascular malformations, platelet lifespan is normal. Destruction of neighboring tissue rarely occurs with hemangiomas occasionally seen are macrotia or maxillary-mandibular hypertrophy. In vascular malformations, especially in those with markedly reduced flow, there is commonly widespread skeletal anomalies and destruction. 5,6

\section{Vascular Tumors Hemangiomas}

Hemangiomas are the most commonly occurring vascular tumors and are the most commonly seen vascular tumors in children, with an incidence of $10 \%$. It is seen three times more often in females compared to males. Other predisposing factors are: light colored skin, premature births, and low birth weight. ${ }^{7}$ Hemangiomas generally occur sporadically, however due to families which show multiple occurrences in subsequent generations, it has been suggested that there may be an autosomal dominant inheritance pattern, specifically a loss of heterozygosity on chromosome 5q31-33.5

$20 \%$ of hemangiomas can be seen at birth, and most times can be visualized in utero, and are termed congenital hemangiomas $(\mathrm{CH})$. $\mathrm{CH}$ are fully formed before birth and unlike infantile hemangiomas $(\mathrm{IH})$ do not proliferate after birth but only have stages of involution. Rapidly involuting congenital hemangiomas $(\mathrm{RICH})$ and non-involuting congenital hemangiomas $(\mathrm{NICH})$ are the two subgroups of $\mathrm{CH}$. $80 \%$ of hemangiomas appear within weeks 1-4 and have stages of proliferation and involution. ${ }^{6}$

The previous terminology scheme for hemangiomas included, superficial capillary hemangioma and deep cavernous hemangioma, those which combined superficial and deep planes were termed mixed capillary cavernous hemangioma. These terms are no longer used and have been replaced by, superficial hemangioma, deep hemangioma (covered by normal skin) and compound hemangioma.8 Hemangiomas appear as erythematous red patches (possibly appearing as a telangiectasia) with a pale halo surrounding it. If the cellular proliferation occurs in the superficial dermis the lesions appear bright red and if it occurs in the deep dermis, the lesions appear blue. Hemangiomas that display a blue-red macule-papule will often become brighter and will display less physical effects of a mass upon entering the involution stage. The complete involution can take up to 5-10 years and the mechanisms of involution (and formation) are not well understood. ${ }^{6}$

Hemangiomas endothelial and placental microvessels co-express a series of tissue-specific markers: GLUT-1, CD14 (Lewis Antigen) CD32 (FcGammaR2) and Mero$\sin$. Those immunohistochemical findings are not found in any other vascular tumor, vascular malformation or in healthy skin. ${ }^{5}$

$60 \%$ of hemangiomas appear in the head and neck region, $25 \%$ in the back region, and $15 \%$ in the extremities. $20 \%$ of all hemangiomas display multiple cutaneous lesions and in those who have more than five lesions, screening for extracutaneous involvement of the gastrointestinal tract, specifically the liver is recommended. History and physical examination are generally sufficient in the diagnosis of hemangiomas with characteristic series of events, rapid growth after birth followed by a static period, ending with a period of regression. Skeletal deformities such as osseous hypertrophy and distortion are rarely seen with hemangiomas (and are more indi- 
cative of lymphatic or venous malformations). MRI with contrast is the preferred method of imaging. ${ }^{9}$

The "watch and wait" ideology as it applies to hemangiomas has all but completely lost its validity. Of all hemangiomas that are not treated and persist to the age of six, only $60 \%$ will heal in an aesthetic or functionally acceptable fashion. The currently accepted treatment ideology is "close observation" which is very different than the "watch and wait" method. There are many benefits to adopting the approach of close observation; correctly diagnosing birthmarks that were misdiagnosed as hemangiomas, allowing for early management of potential complications, decrease in complications (due to regular check-ups) and increased morale with positive psychological state. ${ }^{10}$

One of the most important therapeutic agents is steroid therapy, being used as the first line of treatment in serious and problematic hemangiomas for the past 30 years. The dose-response relationship is $84 \%$, and the best results are achieved if the treatment is started before the age of 6 months. Steroid therapy is especially indicated in mixed hemangiomas, that are in the proliferative stages, and those which are affecting vital organs. The effectiveness of propanolol (a beta-adrenergic antagonist) in treating hemangiomas was serendipitously discovered, and is effective in both the proliferative and involution stages. Having relatively few side effects, it has become the most promising choice of treatment in the cases of $\mathrm{IH}$. Laser treatment can be used (as an adjunct treatment) in superficial hemangiomas to speed up the course of regression and decrease the size of the lesion. If the lesion increases in size despite laser treatment, then the treatment plan must be re-evaluated and an alternate medical treatment should be considered. The advantages of laser treatment are its ease of use and regular treatment intervals of 2-4 weeks. The type of laser used depends on the area it is being used on, the dimensions of the lesion, and the depth of the lesion. Commonly used lasers are; Flash Pump Pulsed Dye Laser (PDL), Nd YAG (Neodymium) and KTP (Potassium Titanyl Phosphate). The effectiveness of laser treatment is $77-100 \%$ with the success rate increasing as the lesi- on size decreases. ${ }^{8}$

Pingyangmycin (Bleomycin A5) is a commonly used and effective alternative for hemangiomas resistant to treatment by Vincristine and Interferon. It is also necessary to consider surgical resection, especially in cases of accessible hemangiomas that can be removed in the course of a single procedure or ulcerated hemangiomas. ${ }^{7}$

\section{Tufted Angiomas}

Tufted angiomas are mostly congenital non-regressed lesions. They usually require pathological diagnosis. Lesions are resistant to medical therapy and may cause local destruction. ${ }^{4}$

\section{Vascular Malformations}

Vascular malformations (VM) are categorized on the basis of their hemodynamic characteristics and the predominantly affected vessel. VM stem from errors during embryogenesis leading to abnormally structured vessels that have normal endothelial turnover. Based on the amount of flow through the vessel, VM are categorized as capillary, venous, lymphatic, arteriovenous and mixed. Since the course of treatment varies with the diagnosis of the subtype, it is imperative that a correct diagnosis be made. ${ }^{1,6}$

\section{Venous Malformations}

Venous Malformations are the most commonly seen VM. Hemodynamically, they are inactive, with relatively low flow and are a malformation of the aggregation of the vascular network. They are generally sporadic, and familial occurrences are seen in less than 1\%. VM are abnormally widened vessels due to the disorganization or absence of smooth muscle around the endothelium. They are generally apparent at birth, presenting as a bluish patch, mark or dilated vein appearance. VM can be localized, generalized, flat, spongy, singular, or multiple in appearance. They can be associated with skin, mucosa, soft tissue, muscle, joints, bones or even the viscera. They are compressible, soft lesions that are often misdiagnosed as hemangiomas, but differ in that unlike hemangiomas, they become progressively worse in varying degrees. Chief complaints of patients with VM are 
swelling and pain. VM can lead to coagulopathies and can contribute to the development of phlebitis. ${ }^{6}$

$\mathrm{MRI}$ is the most useful diagnostic tool for VM, with T1weighted MRI being used for anatomical evaluation and T2-weighted MRI being used to determine the characteristics of low flow lesions (well-defined venous spaces tend to produce a strong signal on T2-weighted MRI). ${ }^{9}$

Treatment options include sclerotherapy and surgery. Sclerotherapy is normally used to reduce the size of the lesion either as preparation for surgical resection or to remove residual tissue post-operatively. The most effective agent in sclerotherapy is ethanol. Aspirin or low molecular weight heparin therapy lowers the risk of thrombotic attack and prevents pain associated with coagulopathy and phlebitis formation. For superficial skin or complex VM, Nd YAG, PDL, Gentile YAG or KTP laser can be used. ${ }^{10}$

\section{Capillary Malformations}

Capillary malformations (CM) are hemodynamically inactive and have low flow lesions that affect skin and/or mucosal capillary networks and are found superficially. Deep tissue invasion is not common unless the lesion is located in the facial area. Examples of $\mathrm{CM}$ are; nevus flammeus, port wine stain, telangiectasias, spider nevus (Spider angioma) or incidental occurrences from related syndromes such as Sturge Weber Syndrome..$^{6-11}$

\section{Port Wine Stain}

Port Wine Stains (PWS) are present in $0.3 \%$ of all births in equal rates in males and females. $45 \%$ of those located on the face are in areas innervated by the trigeminal nerve. PWS generally involve attachment of the mucous membrane to the skin, and in response to increased heat can increase in size and darken in color. As the patient gets older, the surface of the PWS is seen to become irregular, hardened, and take on a nodular appearance. Typically, PWS is seen at birth and increases in size proportionally to the growth of the baby. Since 1980, Flashlamp Pumped Pulse Dye Laser (PDL) has been recommended as the most effective treatment option. Aside from PDL, there are other options such as: $532 \mathrm{~nm}$ KTP (for thinner lesions) 595nm PDL (for associated telangiectasias) and Nd YAG laser (for deep lesions). ${ }^{6-11}$

\section{Cutis Marmorata}

Cutis Marmorata are lesions in children that appear when they are exposed to colder environments (and are not present when they are exposed to warmer environments) and normally go away after puberty. In some patients, however, ectasia in superficial veins can persist for life.

\section{Vascular Angioma}

Vascular Angiomas are commonly seen in the central arterioles of the face and hands, with a radially spreading pattern. When pressure is applied to the center, the lesion is seen to fade. Most vascular angiomas go away after puberty.

\section{Sturge Weber Syndrome}

Sturge Weber Syndrome (SWS) is the most well known syndrome in which PWS are seen. SWS is characterized by facial PWS (specifically in the V1 region) leptomeningeal vascular anomalies and ipsilateral ocular abnormalities. Small and superficial lesion can be treated in a relatively simple manner, however the treatment of deep lesions (especially those that have spread to multiple organs) remains a problem. Complicated cases often require a multi-step treatmen as it is uncommon to achieve successful results with a single step treatment in those cases. ${ }^{6-11}$

\section{Lymphatic Malformations}

Lymphatic malformations (LM) are sporadic lesions often seen in the clinic, most commonly detected in patients when they are around the age of. They are generally composed of improperly formed small vesicles or pouches filled with lymphatic fluid, and are non-tender and soft. $75 \%$ of LMs are detected before the age of 5 and the most common site (36\%) is the head/neck region. LM are the leading cause of macrocheilia, macrognathia and macroglossia. Microcystic LM often lead to clear or hemorrhagic vesicles in the skin or mucosa and can be seen in the thorax, abdomen and bones. Previously called cystic hygromas, macrocystic LMs classically 
manifest as a slightly blue soft mass or lump under the skin. Combined micro and macro cystic forms are rather common. The microcystic form can progress to the macrocystic form following hemorrhage into the cyst or infection. In these cases, analgesic or antibiotic therapy should be considered. 8,11

Ultrasonography and Doppler can be used to detect the multilocular anechoic cysts in macrocystic LM and heterogeneous hypoechoic lesions in microcystic LM. Septal masses and cystic spaces are seen as hypointense with T1 weighted MRI, and appear hyperintense with T2 weighted MRI. Direct puncture allows for analysis of the fluid can help to rule out other cystic lesions and cystic malignancies. Larger lesions can be treated by aspirating the contents of the lesion followed by injection of sclerosing agents under the guidance of fluoroscopy. Laser ablation and surgical treatment are also used as both first line treatment and second line treatment in the cases where sclerotherapy was not sufficient or successful. Nd YAG and Diode Laser Photocoagulation can be used to treat microcystic LM. ${ }^{9}$

\section{Arteriovenous Malformations}

Arteriovenous Malformations (AVM) are hemodynamically active vascular malformations often with rapid flow. AVM are the most aggressive of the malformations, and luckily are not encountered commonly. It consists of a multiple feeding arteries, the core (nidus) and widened draining veins that is pulsatile can be either superficial or deep. Although present in many births, AVM are generally not detected until puberty and are not known to reg- ress. In infants, AVM are not pulsatile, do not contain a rapid flow, and do not radiate heat to their surroundings, they are generally misdiagnosed as hemangiomas. Ultrasonography and Doppler can detect AVM shunts, and MRI will show the damaged vessel network. 4,7

Endovascular embolization and surgical resection are the main treatment protocols. The palliative (arterial embolization) complication control (ulcers, hemorrhaging, lytic lesions in the bone) or curative (broad surgical resection/ reconstruction following embolization) treatments for AVM is always a challenging process. Partial resection and proximal vessel occlusion are contraindicated in surgical treatment options since any residual tissue left behind has the tendency to grow faster than the originally resected tissue did. ${ }^{12-13}$

\section{Conclusion}

Even when functional problems are absent, birthmarks can cause psychological trauma to the patient and their families. Therefore a correct diagnosis is the first step in treatment. The correct nomenclature, classification, and most current treatment modality is not always known to those physicians who are not experts in the field. In these cases, it is of utmost importance for the patients to be referred to doctors or clinics specializing in this area so that the diagnosis-treatment algorithm can be properly applied. As such, the guidelines will be periodically reviewed and updated to reflect the most current knowledge regarding the topic, with the goal of providing the best possible treatment outcomes for affected patients. 


\section{References}

1. Mulliken JB, Glowacki J. Hemangiomas and vascular malformations in infants and children: A classification based on endothelial characteristics. Plast Reconstr Surg 1982; 69:412-22.

2. Jackson IT, Carreño R, Potparic Z, Hussain K. Hemangiomas, vascular malformations, and lymphovenous malformations: Classification and methods of treatment. Plast Reconstr Surg. 1993; 91:1216-30

3. Arneja JS. Where have we arrived in the care of vascular anomalies a generation after Mulliken's classification system? Can J Plast Surg. 2011; 20:65.

4. Kollipara R, Odhav A, Rentas KE, et al. Vascular anomalies in pediatric patients: updated classification, imaging and therapy. Radiol Clin North Am. 2013; 51:659-72.

5. Aboutalebi A, Jessup CJ, North PE et al. Histopathology of vascular anomalies. Facial Plast Surg. 2012; 28(6):545-53. doi: 10.1055/s-0032-1329929.

6. Lee BB, Laredo J. Classification of congenital vascular malformations: the last challenge for congenital vascular malformations. Phlebology. 2012; 27:267-9.

7. Zheng JW, Zhou Q, Suen JY. Treatment guideline for hemangiomas and vascular malformations of the head and neck. Head Neck. 2010; 32:1088-98.

8. Eivazi B, Werner JA. Management of vascular malformations and hemangiomas of the head and neck—an update. Curr Opin Otolaryngol Head Neck Surg. 2013 21:157-63. doi: 10.1097/ MOO.0b013e32835e15a9.

9. Chaudry MI, Manzoor MU, Turner RD et al. Diagnostic imaging of vascular anomalies. Facial Plast Surg. 2012; 28:563-74. doi: 10.1055/s-0032-1329931.

10. Zheng JW, Mai HM, Suen JY. Guidelines for the treatment of head and neck venous malformations. Int $\mathrm{J}$ Clin Exp Med. 2013,22;6:377-89.

11. Behr GG, Johnson C. Vascular anomalies: hemangiomas and beyond--part 1, Fast-flow lesions. AJR Am J Roentgenol. 2013; 200:414-22

12. Behr GG, Johnson CM. Vascular anomalies: hemangiomas and beyond--part 2, Slow-flow lesions. AJR Am J Roentgenol. 2013; 200:423-36

13. Abramowicz S, Padwa BL. Vascular anomalies in children. Oral Maxillofac Surg Clin North Am. 2012 Aug;24:443-55 